\title{
Comparing the performance of Organic-inorganic hybrid tandem multijunction solar cells of different organic bulk thicknesses
}

\author{
Abdolnabi Kosarian ${ }^{1}$, Mehrdad Kankanan ${ }^{2}$ e Mohamad Ali Khalafi ${ }^{3}$ \\ 1,3 Shahid Chamran University of Ahvaz \\ ${ }^{2}$ Shahid Chamran University of Ahvaz, eng_kankanan@yahoo.com
}

\begin{abstract}
In this study, J-V curves of a-Si:H/PCPDTBT:PC70BM hybrid tandem solar cells were simulated using a modified drift-diffusion model, and the influence of the thickness of the organic blend layer was investigated. The results of the simulations were compared with experimental data from literature.

It is shown that as the thickness of the blend layer increases, the fill factor and the voltage corresponding to maximum power point decrease whereas the maximum power point and the short circuit current density of solar cell increase up to thicknesses of $60 \mathrm{~nm}$ and 138 $n m$ respectively. Finally, the modified organic solar cell was used as second sub-cell and the power conversion efficiency increased from $1.90 \%$ to $2.1 \%$ in simulation.
\end{abstract}

Keywords: hybrid tandem solar cell, Koster method, blend layer thickness, PCE. 


\section{Introduction}

$\mathrm{I}$ $\mathrm{n}$ recent years, there has been an expansion of interest in organic solar cells due to their low cost process, high absorption coefficient and green environment technologies. Because of their narrow absorption spectrum as compared with crystal materials, their relatively low electric constant and suffering from their stability, the organic-inorganic hybrid structure has been proposed.

In these devices, the inorganic upper sub-cell absorbs the UV photon from the sun spectrum significantly reduces degeneration of organic lower sub-cell . Additionally, they increase the power conversion efficiency due to their tandem structure. Hydrogenated amorphous silicon (a$\mathrm{Si}: \mathrm{H})$ is one of the candidate materials for upper sub-cell because of its relatively high open circuit voltage $(V o c)$ and fill factor (F.F).

Fig. 1. shows the configuration of the organicinorganic hybrid tandem solar cell. As shown in Fig. 1, the a-Si:H first sub-cell is deposited on 50 $\mathrm{nm}$ ITO coated glass. The thicknesses of the aSi:H layers are $5 \mathrm{~nm}, 120 \mathrm{~nm}$, and $25 \mathrm{~nm}$ for $p^{+}, i$, $n^{+}$layers, respectively. A $5 \mathrm{~nm}$ intermediate layer made of $\mathrm{MoO}_{3}$ (Aldrich) was applied between the sub-cells as an efficient hole transporting layer. The $70 \mathrm{~nm}$ bulk PCPDTBT:PC70BM organic layer was used as a second sub-cell. And finally $20 \mathrm{~nm} \mathrm{TiO} 2$ electron transport layer and $100 \mathrm{~nm}$ Aluminum contact were applied.

The employed simulation is based on a modified drift-diffusion model, taking into account results from microscopic modeling. The model can be used to describe the hopping nature of charge transport and the bimolecular Langevin recombination in organic material. To account for disorder in amorphous sub-cell, a Gaussian broadened density of state and FermiDirac statistic with a generalized Einstein relation are applied.

Transmittance of the a-Si:H $p^{+}-i-n^{+}$layer and absorbance of the PCPDTBT:PC ${ }_{70} \mathrm{BM}$ blend film are considered as mentioned in.

\section{Numerical simulation}

There are several models for the simulation of semiconductor devices. Drift-diffusion model (DDM) is one of the most widely used among them, which includes the following three equations:

$$
\begin{aligned}
& \nabla .(\varepsilon V(r))=q(n(r)-p(r)+D o p(r)) . \\
& \nabla . J_{n}(r)=-q U(r) . \\
& \nabla . J_{p}(r)=q U(r) .
\end{aligned}
$$

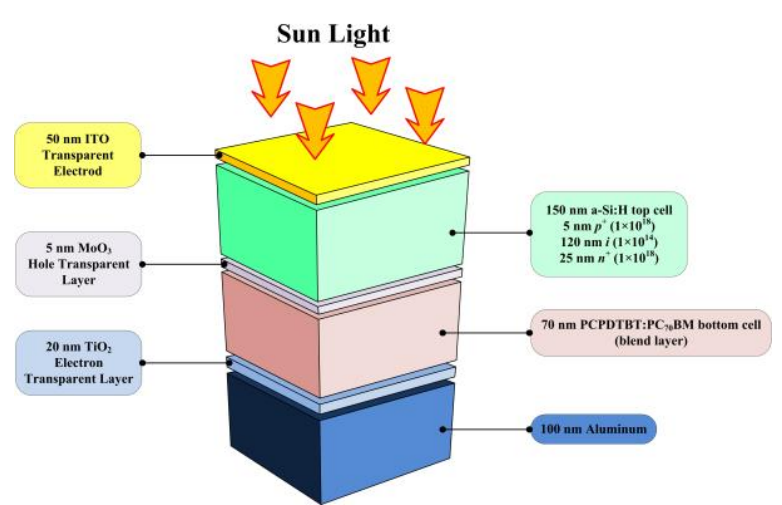

Fig. 1 : Device Configuration [4].

where $V$ is the electrostatic potential, $n$ is the electron density, $p$ is the hole density, Dop is the doping profile, $J_{n}$ and $J_{p}$ are the electron and hole current density, $U$ is the net generationrecombination rate of conduction electrons and holes, $q$ is the electron charge, and $\varepsilon$ is the dielectric permittivity.

In 1964, Gummel proposed a self-consistent method for solving these equations. In this algorithm, the Poisson's equation is solved for an initial condition for the carrier density. Then, the current continuity equation for the electron and hole are solved for the potential obtained from Poisson's equation. Poisson's equation will be resolved again for the new values obtained from the current continuity equation. This trend continues until a desired error is achieved.

Gummel's method is based on a highly effective decoupling algorithm which is widely used in crystalline semiconductor device simulation. Because the desired solar cell structure consists of a hydrogenated amorphous silicon upper sub-cell and an organic lower subcell, we used the adapted Gummel's method 
proposed by Kemp for amorphous semiconductors and Koster's model for organic semiconductors which are described below.

\subsection{Adapted Gummel method}

There are always defects in the structure of amorphous materials that can be caused by dangling-bonds in the surface or the presence of impurities within the bulk. The presence of these defects will change the electrical properties of the device because of their trap levels in the bandgap and changes in the carrier density of conduction and valance bands.

These changes were modeled by Kemp. They imported a trapped carrier density into the left hand side of Poisson's equation and modified the initial and boundary conditions which is described as follows:

$$
\begin{aligned}
& \nabla .(\varepsilon V(r))=q\left(n(r)-p(r)+\operatorname{Dop}(r)+Q_{\text {trap }}\right) \\
& Q_{\text {trap }}=N_{D . t r a p}^{+}-N_{\text {A.trap }}^{-}
\end{aligned}
$$

where $N^{+} D$.trap and $N^{+}$D.trap are the densities of ionized donor-like and acceptor-like traps respectively. Moreover, they used SRH recombination model to describe the effect on recombination states.

\subsection{Koster's model}

Many researchers applied models valid for inorganic devices to organic devices. However, the difference between the generation and recombination processes in organic and inorganic materials reduces the accuracy and validity of the previous works. In 2007 Koster proposed a model for explaining the electrical behavior of a bulk Heterojunction organic solar cell. In his model, the blend layer of two organic materials is equivalent to a single material whose bandgap is the same as the effective bandgap described by Vandewal as follows.

$E_{\text {eff }}=\frac{1}{e}\left[H O M O_{\text {Donor }}-L U M O_{\text {Acceptor }}\right]-0.43 \mathrm{~V}$

where $E_{\text {eff }}$ is the effective bandgap, HOMODonor is the highest occupied molecular orbit and LUMOAcceptor is the lowest un-occupied molecular orbit of acceptor organic material.
The model also accounts for bimolecular Langevin recombination. Equation (6) is the mathematical description of Koster's exciton change rate, where $G(x)$ is the one-dimentional photogeneration rate of excitons and described as Equation (7).

$\frac{d X}{d t}=G-k_{f} X-k_{d i s s} X+R$
$G(x)=G_{0} e^{-x}$.
$R=q \frac{\min \left(\mu_{n}, \mu_{p}\right)}{\varepsilon}\left(n p-n_{i}^{2}\right)$.

where $R$ is the bimolecular Langvin recombination, $\mu_{n}$ and $\mu_{p}$ are electron and hole mobility, $n_{i}$ is the intrinsic carrier concentration, $k_{f}$ is the bound pair decay rate, $k_{\text {diss }}$ is the exciton dissosiation rate constant and $X$ is the density of electron-hole pairs.

\section{Simulation results and discussion}

The accuracy of the simulation depends on the accuracy of the material parameters used and the device model. Table 1 and Table 2 show the parameters used for the simulation of the hybrid tandem solar cell illustrated in Fig. 1. In addition, for simulation of a solar cell, it may be illuminated by a constant wavelength of light or a complex spectrum such as Air Mass Zero (AM0) or AM1.5 spectrum. Since in article [4] the current density-voltage $(J-V)$ curves was measured under light intensity of $100 \mathrm{~mW} / \mathrm{cm}^{2}$, the AM1.5G spectrum was used in this simulation.

Table 1. Electrical properties of PCPDTBT:PC ${ }_{70} \mathrm{BM}[8]$.

\begin{tabular}{|c|c|c|}
\hline Model Parameter & symbol & Value \\
\hline Effective & $E_{g a p}$ & $1.1(\mathrm{e} . \mathrm{V})$ \\
\hline Electron mobility & $\mu_{n}$ & $7 \times 10^{-4}\left(\mathrm{~cm}^{2} / \mathrm{Vs}\right)$ \\
\hline Hole mobility & $\mu_{p}$ & $4 \times 10^{-4}\left(\mathrm{~cm}^{2} / \mathrm{Vs}\right)$ \\
\hline $\begin{array}{c}\text { Effective density } \\
\text { of states }\end{array}$ & $N_{C}$ & $2.5 \times 10^{25}\left(\mathrm{~cm}^{-3}\right)$ \\
\hline Generation rate & $G_{0}$ & $9.5 \times 10^{21}\left(\mathrm{~cm}^{-3}\right)$ \\
\hline $\begin{array}{c}\text { Relative } \\
\text { dielectric }\end{array}$ & $\varepsilon_{r}$ & 3.4 \\
\hline $\begin{array}{c}\text { Initial bound pair } \\
\text { distance }\end{array}$ & $a$ & $1.3(\mathrm{~nm})$ \\
\hline Bound pair decay & $k_{f}$ & $3 \times 10^{6}\left(\mathrm{~s}^{-1}\right)$ \\
\hline
\end{tabular}


Table 2. Electrical properties of a-Si:H.

\begin{tabular}{|c|c|c|}
\hline Model Parameter & symbol & Value \\
\hline Effective bandgap & $E_{\text {gap }}$ & $1.8(\mathrm{e} . \mathrm{V})$ \\
\hline Electron mobility & $\mu_{n}$ & $20\left(\mathrm{~cm}^{2} / \mathrm{Vs}\right)$ \\
\hline Hole mobility & $\mu_{p}$ & $2\left(\mathrm{~cm}^{2} / \mathrm{Vs}\right)$ \\
\hline $\begin{array}{c}\text { Effective density } \\
\text { of states }\end{array}$ & $N_{C}$ & $2.5 \times 10^{20}\left(\mathrm{~cm}^{-3}\right)$ \\
\hline Generation rate & $G_{0}$ & $9.5 \times 10^{21}\left(\mathrm{~cm}^{-3}\right)$ \\
\hline
\end{tabular}

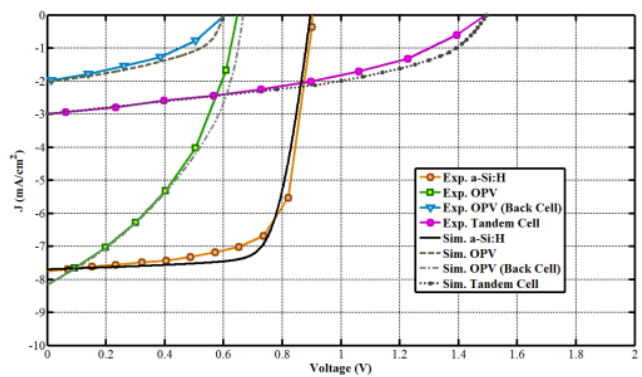

Fig. $2: J-V$ curves of single-junction and tandem hybrid multijunction solar cells. The solid lines show experimental data and the dotted lines show simulation results

Results obtained from simulation using modified DDM for single-junction and tandem multijunction hybrid solar cell are shown in Fig. 3. The solid lines show the experimental data extract using WebPlotDigitizer which is a free web-based application and the dotted line shows the simulation results for the corresponding cells. The corresponding Photovoltaic parameters of solar cells are listed in Table 3. As can be seen, there is a very good correlation between the experimental data and the results of the simulation.

After the necessary accuracy of the simulation was observed, we used this model to optimize the thickness of the active layer of bulk organic cell.

Although there was a slight difference between the experimental data and the simulation results, this would not affect the process of optimizing the blend layer in the organic second cell, because this difference is constant and exists in all results.

To study the effect of the thickness of the blend layer on the outputs of the organic cell and subsequently the organic-inorganic tandem hybrid cell, the thickness of the active layer of the PCPDTBT:PC $70 \mathrm{BM}$ cell was changed from 10 $\mathrm{nm}$ to $400 \mathrm{~nm}$, and the major output parameters as the open circuit voltage, the short circuit current, the fill factor, the current and the voltage corresponding to maximum power point, and the maximum output power were measured. The results are shown in Fig. 3.

It is observed from Fig. 3 that the open circuit voltage $(V o c)$ is almost constant with the variation in thickness such that it initially decreases slightly with the increase in thickness but then remaining constant. Needless to say, the open circuit voltage is directly proportional to the effective band gap of the device so the $V_{O C}$ remains predictably constant. Due to the bimolecular recombination and the bound pair decay rate, the fill factor (F.F) and voltage $V_{m}$ corresponding to the maximum power point show a decreasing trend with the increase in thickness.

The short circuit current density (Jsc) increases up to a thickness of $138 \mathrm{~nm}$ with increased thickness of active layer, but it will have a downward trend thereafter. Thus, it is predictable that the maximum output power should have a maximum point. This point should be placed at a thickness of $60 \mathrm{~nm}$. Like the short circuit current, the maximum current $\left(J_{m}\right)$ initially increases by increasing the thickness of the active region to $100 \mathrm{~nm}$, and then it decreases after the thickness of active layer is increased.

In order to increase the short circuit current density of the cell, (considering that the open circuit voltage is almost constant by changing the thickness of the active region) $138 \mathrm{~nm}$ thick is assumed for the active region. The simulation results for the modified hybrid tandem solar cells with $138 \mathrm{~nm}$ thickness of organic blend layer are shown in the last row of Table 3. As can be seen, the short circuit current density and PEC increase from $2.98\left(\mathrm{~mA} / \mathrm{cm}^{2}\right)$ and $1.9(\%)$ to 3.62 $\left(\mathrm{mA} / \mathrm{cm}^{2}\right)$ and $2.1(\%)$ respectively. 
Table 3 : The photovoltaic results for single-junction and tandem hybrid multijunction solar cells. The bold numbers represent experimental data from and the other columns are related to simulation results.

\begin{tabular}{|c|c|c|c|c|c|c|c|c|c|}
\hline \multirow{2}{*}{\multicolumn{2}{|c|}{ Devices }} & \multicolumn{2}{|c|}{$\mathrm{V}_{\mathrm{OC}}(\mathrm{V})$} & \multicolumn{2}{|c|}{$\mathrm{J}_{\mathrm{SC}}\left(\mathrm{mA} / \mathrm{Cm}^{2}\right)$} & \multicolumn{2}{|c|}{ F.F (\%) } & \multicolumn{2}{|c|}{ PCE (\%) } \\
\hline & & Exp. & Sim. & Exp. & Sim. & Exp. & Sim. & Exp. & Sim. \\
\hline \multirow{3}{*}{$\begin{array}{l}\text { Single- } \\
\text { junction }\end{array}$} & OPV & 0.658 & 0.660 & 8.08 & 8.10 & 41 & 40.06 & 2.16 & 2.18 \\
\hline & OPV (back cell) & 0.609 & 0.610 & 2.00 & 2.02 & 40 & 40.50 & 0.48 & 0.50 \\
\hline & $\mathrm{a}-\mathrm{Si}: \mathrm{H}$ & 0.906 & 0.900 & 7.71 & 7.76 & 71 & 71.80 & 4.93 & 5.00 \\
\hline \multicolumn{2}{|c|}{ Tandem hybrid multijunction } & 1.499 & 1.495 & 2.98 & 2.98 & 41 & 43 & 1.84 & 1.90 \\
\hline \multicolumn{2}{|c|}{ Modified tandem Cell ${ }^{* *}$} & & 1.475 & & 3.62 & & 40 & & 2.10 \\
\hline
\end{tabular}

* PCE : Power Conversion Efficiency

** Modified tandem hybrid solar cell with $138 \mathrm{~nm}$ thickness of the second organic blend layer

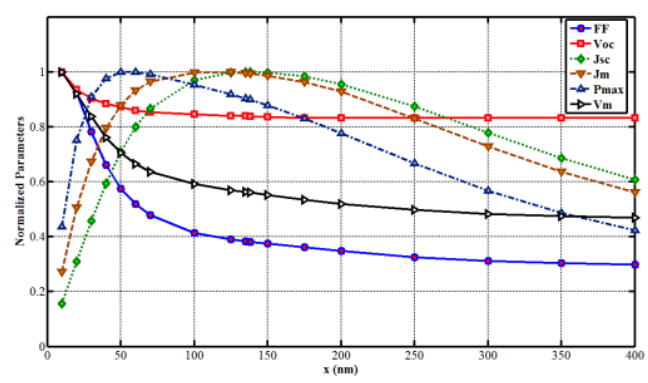

Fig. 3 : Performance parameters as a function of blend thickness of single organic solar cell

\section{Conclusion}

We have simulated an organic-inorganic hybrid tandem multijunction aSi:H/PCPDTBT:PC70BM solar cell using a modified drift-diffusion model. The results of simulation were compared with experimental data. The dependence of the short circuit current density, the open circuit voltage, the fill factor, the current density and voltage corresponding to maximum power point, and the maximum output power on the thickness of the active region of organic sub-cell were quantified and the optimum thickness of the blend layer for the maximum short circuit current density was determined.

Finally the modified hybrid tandem solar cell with $138 \mathrm{~nm}$ thickness of the organic active layer was simulated and it was observed that the short circuit current density and PEC increased from $2.98\left(\mathrm{~mA} / \mathrm{cm}^{2}\right)$ and $1.9(\%)$ to $3.62\left(\mathrm{~mA} / \mathrm{cm}^{2}\right)$ and $2.1(\%)$ respectively.

\section{References}

Wei, Q., et al. (2014). High efficiency organic/a-si hybrid tandem solar cells with complementary light absorption. Journal of Materials Chemistry A, vol. 2, no. 37, p. 1530315307.2014.

Brabec, C. J., et al. (2001). Origin of Open Circuit Voltage of Plastic Solar Cells. Adv. Funct. Mater, vol. 11, no. 5, p. 374-380. 2001.

Wright, M., Uddin, A., (2012). Organic inorganic hybrid solar cells: A comparative review. Solar Energy Materials $\mathcal{E}$ Solar Cells, vol. 107, p. 87-111. 2012.

Kim, T., et al. (2011). Organic-inorganic hybrid tandem multijunction photovoltaics with extended spectral response. Applied Physics Letters, vol. 98, no. 18, p. 183503 (1)- 183503 (3). 2011.

Koster, L. J. A., Smits, E. C. P., Mihailetchi, V. D., Blom, P.W. M., (2005). Device model for the operation of polymer/fullerene bulk Heterojunction solar cells. Phys. Rev. B, vol. 72, no. 8, p. 085205 (1) - 085205 (9).2005.

Pattnaik, S., Teng Xiao, Shinar, R., Shinar, J., Dalal, V.L., (2013) Novel Hybrid Amorphous/Organic Tandem Junction Solar Cell. Photovoltaics, IEEE Journal of , vol.3, no.1, pp.295,299, Jan. 2013.

Kemp, K., Tannous, C. G., Meunier, M., (1988). Amorphous Silicon Device Simulation by an Adapted Gummel Method. IEEE Transaction 
on Electron Devices, vol.35, no.9, pp.1510, 10513. 1988.

Moet, D. J. D., et al. (2010). Enhanced dissociation of charge-transfer states in narrow band gap polymer:fullerene solar cells processed with 1,8-octanedithiol. Applied Physics Letters, vol.96, no.21, pp.213506 (1)213506 (3). 2010.

Kosarian, A., Jelodarian, P., (2011). Optimization and characterization of advanced solar cells based on thin-film a-Si:H/SiGe heterostructure. Electrical Engineering (ICEE), 2011 19th Iranian Conference on , vol., no., pp.1,5, 1719. 2011.

Gummel, H. K., (1964). A Self -Consistent Iterative Scheme for One-Dimensional Steady State Transistor Calculations. IEEE. Trans. Electron Devices, Vol.11, No. 10, pp. 455-465. 1964.

Servaites, J. D., Ratner, M. A., Marks, T. J., (2011).Organic solar cells: A new look at traditional models. Energy Environ. Sci., Vol.4, No. 11, pp. 4410-4422. 2011.

[Online], http://arohatgi.info/WebPlotDigitizer/>, [14 Aug 2015].

Saradhi, A., Prabhakara Rao, B.V.V.S.N., (2014). Comparison of performance in P3HT:PCBM bulk heterojunction organic solar cells of different thickness. Electronics, Computing and Communication Technologies (IEEE CONECCT), 2014 IEEE International Conference on, vol., no., pp.1-3. 2014.

Junsangsri, P., Lombardi, F., (2010). Modelling and extracting parameters of organic solar cells. Electronics Letters, vol.46, no.21, pp.14621464. 2010.

Vandewal, K., et al. (2008). The relation between open-circuit voltage and the onset of photocurrent generation by charge-transfer absorption in polymer:fullerene bulk Heterojunction solar cells. Advanced Functional Materials, Vol.18, No. 14, pp. 20642070. 2008. 